\title{
A EXPERIÊNCIA RECENTE Do PRONAF EM PERNAMbUCO: UMA ANÁLISE POR MEIO DE PROPENSITY SCORE
}

\author{
André Matos Magalhães ${ }^{\S}$ \\ Raul Silveira Neto \\ Fernando de Mendonça Dias ${ }^{\dagger}$ \\ Alexandre Rands Barros
}

\begin{abstract}
RESUMO
O objetivo desse trabalho é avaliar a experiência do PRONAF entre os agricultores do Estado de Pernambuco. São utilizados dados sobre aproximadamente 4.500 agricultores familiares desse Estado, coletados em 2001, divididos entre beneficiários do programa e um grupo de controle. Os resultados são apresentados para a amostra total, excluídos os assentados, e por grupo do PRONAF (B, C e D). As estimativas são corrigidas para a existência de viés de participação com a utilização do método de Propensity Score. Os resultados indicam que o PRONAF mostrou-se pouco eficaz em Pernambuco até esse período. Quando as diferenças de características e probabilidades de participação do programa são consideradas, os resultados indicam que o programa não tem gerado um impacto significativo para o público-alvo em Pernambuco.
\end{abstract}

Palavras-chave: agricultura familiar, PRONAF, propensity score.

\begin{abstract}
The main goal of the paper is to evaluate the experience of PRONAF on the farmers of the state of Pernambuco. The data used include information on, approximately, 4.500 farmers from this State. The results are presented for the whole sample, excluding the "assentados", and by groups (B, C e D). The estimates are corrected for the existence of participation bias through the use propensity score method. The effectiveness of the program is also considered in the analysis. When the differences of characteristics and probabilities of participation are factored in, the main findings point that, although effective, the program has not shown significant impacts on the considered state.
\end{abstract}

Key Words: family farm, PRONAF, propensity score.

JEL classification: Q12.

$\S \quad$ Ph.D. em economia pela Universidade de Illinois em Urbana-Champaign. Professor do PIMES-UFPE, pesquisador do CNPq e pesquisador associado do Regional Economics Applications Laboratory (REAL).E-mail: magalhaes@decon.ufpe.br. Endereço para contato: Rua Padre Roma, 652, apt. 101. Parnamirin - Recife - Pernambuco - CEP: 52060-060.

a Doutor em economia pela USP. PIMES-UFPE e pesquisador do CNPq. E-mail: rau.silveira@uol.com.br.

$\dagger$ Doutor em Economia pelo PIMES-UFPE. Faculdade Boa Viagem. E-mail: fmd@datametrica.com.br.

† Ph.D. em economia pela Universidade de Illinois em Urbana-Champaign. PIMES-UFPE. E-mail: Alexandre.rands@datametrica.com.br.

Recebido em agosto de 2004. Aceito em novembro de 2005. 


\section{INTRODUÇÃO}

O Programa Nacional de Fortalecimento da Agricultura Familiar (PRONAF) é uma das principais políticas do País na área social e na de agricultura desde a segunda metade da década de 1990, tendo firmado, em um período de quatro anos (entre 1998 e 2001), 2,543 milhões de contratos em todo o País e gasto mais de 6,9 bilhões de reais. Os gastos do programa representaram 0,94\% do Orçamento Federal em 2000, ou aproximadamente 13,37\% dos gastos sociais do Governo Federal naquele ano.

O PRONAF tem como um de seus principais objetivos a redução da pobreza que atinge os agricultores familiares no Brasil. O programa busca assegurar o acesso ao crédito barato a pequenos produtores, além de integrá-los a outras políticas de desenvolvimento rural, como suporte ao desenvolvimento de infra-estrutura e assistência técnica.

O objetivo deste trabalho é avaliar a experiência do PRONAF no Estado de Pernambuco. Com um perfil distinto do encontrado no Sul do País, onde, segundo Cardoso e Ortega (2000), verificam-se efeitos positivos para o PRONAF, os agricultores familiares nesse Estado estão sujeitos às mesmas regras para concessão de financiamentos. A região Sul concentrava, no final da década de 1990, a maior parcela dos financiamentos, mas nesta região os agricultores familiares utilizavam os recursos em cadeias produtivas já existentes, como no fumo, no milho e na soja. Nas demais regiões do País o nível de desenvolvimento da agricultura familiar não é similar, particularmente no Norte e no Nordeste, onde esta é tipicamente uma agricultura de subsistência. Sendo o objetivo do PRONAF reduzir os níveis de pobreza e desenvolvimento no campo, é em Estados como Pernambuco que ele deveria buscar ser mais efetivo.

Para proceder a esta análise serão utilizados dados sobre aproximadamente 4.500 agricultores familiares de Pernambuco em 2001, divididos entre beneficiários e não-beneficiários do programa (grupo de controle). Será analisada a efetividade do programa por meio dos seus impactos sobre as variáveis selecionadas. Os impactos são considerados, separadamente, por grupos do PRONAF (B, C e D) e para o total da amostra. As estimativas são corrigidas para a existência de viés de participação com a utilização do método de Propensity Score.

No que segue, apresenta-se, na seção 2, a experiência recente do PRONAF. Na seção 3 são descritos os dados que serão utilizados nas análises. Na seção 4, é apresentada a metodologia aplicada neste estudo. Na seção 5, discute-se a eficácia do programa a partir de uma análise econométrica. Na última seção são apresentados os comentários finais.

\section{A EXPERIÊNCIA RECENTE do PRONAF}

O Programa Nacional de Fortalecimento da Agricultura Familiar, mais conhecido como PRONAF, tem como objetivo promover o desenvolvimento sustentável do segmento rural constituído pelos agricultores familiares, com vistas a propiciar o aumento da capacidade produtiva, a geração de empregos e a melhoria de renda desses agricultores. O PRONAF foi criado em 1996, tendo como fontes de recursos o Fundo de Amparo ao Trabalhador (FAT), os Fundos Constitucionais de Desenvolvimento (FNO, FNE e FCO) e a exigibilidade bancária. O programa foi posteriormente transferido para a esfera de ações do Ministério de Política Fundiária em 1999, a partir do qual o Conselho Nacional de Desenvolvimento Rural passou a integrar as atividades do PRONAF e do

1 Esses números não incluem os gastos com a Previdência Social, pois esta tem financiamento de fontes diretas. 
INCRA. Atualmente, o PRONAF encontra-se subordinado ao Ministério do Desenvolvimento Agrário (MDA).

A motivação inicial para a criação do PRONAF é dada pela dimensão que a agricultura familiar ocupa na economia brasileira e pela dificuldade, por parte dos agricultores que integram essa categoria, de obtenção de crédito para a produção. Esses agricultores familiares são definidos, de acordo com as especificações do programa, como aqueles que possuem até quatro módulos fiscais, têm até dois empregados permanentes e obtêm pelo menos $80 \%$ da renda familiar da atividade desenvolvida na propriedade. De acordo com o MDA, 84\% dos estabelecimentos agropecuários são familiares, o que se traduz em $80 \%$ do emprego, $40 \%$ do valor da produção agropecuária e de um volume de alimentos superior a $50 \%$ do total produzido no País. ${ }^{2}$

O programa combina, no seu funcionamento, quatro instrumentos básicos: (i) oferta de linhas de crédito especiais, a taxas de juros preferenciais, e com menores exigências de garantias do que as que se encontram no mercado; (ii) financiamento, a fundo perdido, de investimentos em infra-estrutura econômica de suporte aos pequenos produtores; (iii) oferta de assistência técnica aos beneficiários do programa; (iv) oferta de oportunidades de capacitação profissional.

Com exceção dos créditos de infra-estrutura, que são repassados a municípios selecionados ${ }^{3}$ e devem ser empregados na melhoria da infra-estrutura para os agricultores familiares, todos os demais recursos se destinam ao produtor (diretamente ou via cooperativas) e para a assistência técnica.

No período de 1996 a 2000, o número de contratos de crédito assinados cresceu a uma taxa de $27,2 \%$ ao ano, enquanto que o volume de recursos aplicados aumentou a uma razão de $34 \%$ ao ano. Em 2000, foram contraídos aproximadamente $\mathrm{R} \$ 1,8$ bilhão em empréstimos pelos agricultores, volume ainda inferior a $50 \%$ do orçamento do programa para esse ano. De fato, este problema perdurou até 2003, ano a partir do qual se registrou um crescimento de $44 \%$ no número contratos e chegou-se à marca dos $\mathrm{R} \$ 5,4$ bilhões em créditos.

Em termos operacionais, os agricultores que são beneficiários de créditos do PRONAF são divididos em quatro grupos (A, B, C e D) que são classificados, basicamente, segundo sua renda bruta anual e o montante financiado, excetuando-se o grupo A, que é composto exclusivamente pelos assentados do Programa Nacional de Reforma Agrária independentemente de sua faixa de renda. Em relação aos beneficiários dos demais grupos, o PRONAF classificava-os, para o período compreendido entre 1998 e 2000 (anos para os quais os dados foram coletados), de acordo com os seguintes intervalos de renda bruta anual: ${ }^{5}$ até $\mathrm{R} \$ 1.500,00$ para os agricultores do grupo $\mathrm{B}$; de $\mathrm{R} \$$ $1.500,00$ a $\mathrm{R} \$ 8.000,00$ para os agricultores do grupo C; e de $\mathrm{R} \$ 8.000,00$ a $\mathrm{R} \$ 27.500,00$ para os agricultores do grupo D. A análise realizada a seguir levará em consideração esses grupos de renda.

Apesar dos objetivos almejados e do grande esforço despendido pelo MDA na elaboração das regras do PRONAF, as avaliações recentes não têm sido conclusivas no sentido de se verificar os efeitos positivos de sua implementação e, em certos casos, há mesmo evidências de efeitos negativos. De acordo com resultados encontrados no relatório da FECAMP (2002), por exemplo, foram encontrados efeitos significativos e negativos sobre a renda dos domicílios beneficiados para os Estados de Alagoas, Bahia, Ceará, Espírito Santo, Maranhão, Minas Gerais, Rio Grande do Sul e Santa

2 Dados atualmente divulgados no site do PRONAF e oriundos do estudo da FAO/INCRA (2000), que é baseado nos dados do Censo Agropecuário de 1996.

3 Os critérios de seleção dos municípios privilegiam os que possuem grande número de agricultores familiares e apresentam condições econômicas precárias, tendendo assim a selecionar, em geral, os municípios mais pobres em cada Estado.

4 O programa de crédito direto ao produto representa $90 \%$ dos recursos totais.

5 A renda bruta é a renda total do domicílio excetuando-se aposentadorias e pensões. 
Catarina. Tal como este artigo, o relatório da FECAMP baseou-se em uma pesquisa de campo com 1.994 domicílios nos oito Estados supracitados.

Utilizando dados secundários e hipóteses heterodoxas sobre a diferenciação entre PRONAF e controle, Feijó (2003) encontra resultados similares em relação à produtividade entre beneficiários e não-beneficiários no Brasil, embora este autor aponte alguma evidência de crescimento no grupo PRONAF para períodos muito recentes. Utilizando dados primários para a Bahia, em 2001, Couto Filho e Cerqueira (2002) afirmam que não há evidências conclusivas acerca de diferenciações na renda entre beneficiários e não-beneficiários do PRONAF que tenham as mesmas características e, corroborando o indicado por Feijó (2003), encontram evidências de crescimento muito recente de produtividade, principalmente devido à natureza dos financiamentos, que muitas vezes estão condicionados à aquisição máquinas, equipamentos e outros investimentos. Para Couto Filho e Cerqueira (2002), "praticamente não houve aplicação do PRONAF - crédito em atividades agrícolas não-tradicionais, indo de encontro ao defendido na concepção do próprio programa." (p. 53). Este resultado contrasta com os encontrados por Cardoso e Ortega (2000), que comprovam a existência de efeitos positivos do PRONAF na região Sul do Brasil, onde os recursos se mostram altamente eficientes ao se integrarem às cadeias já existentes no fumo, no milho e na soja.

\section{OS DADOS}

Uma forma de avaliar o desempenho do PRONAF é comparar o desempenho dos agricultores que receberam os benefícios em duas situações distintas: antes e depois do recebimento dos créditos. Com essa informação, e com a utilização de um grupo de controle (agricultores que não teriam recebido os benefícios) talvez fosse possível verificar se o programa estaria causando efeito sobre os agricultores. ${ }^{6}$

Como para este estudo não se dispõe de informações sobre o desempenho dos agricultores antes do recebimento dos créditos do PRONAF, as comparações antes e depois do PRONAF não são possíveis. Dispõe-se, todavia, de informações sobre agricultores que receberam crédito durante os anos de 1998 a 2001 e dos seus desempenhos agrícolas no ano de 2001. De forma mais específica, os dados utilizados neste trabalho são provenientes de uma pesquisa de campo realizada pela FADE-UFPE, com agricultores familiares de Pernambuco, durante os meses de agosto a novembro de 2001. Foram aqui utilizadas informações sobre aproximadamente 4.500 pequenos agricultores, sendo dois terços da amostra constituída por beneficiários do PRONAF e a restante formada por não-beneficiários, mas com perfil socioeconômico semelhante aos primeiros e residindo na mesma região dos beneficiários. Este segundo grupo foi utilizado como grupo de controle e servirá de base para comparações com os beneficiários. A Tabela 1, a seguir, apresenta as principais características destes dois grupos.

As entrevistas foram realizadas em mais de 60 municípios localizados no Estado, o que permitiu a inclusão de agricultores das várias microrregiões que produzem diversos produtos financiados pelo programa. Destes 60 municípios, 35 são receptores dos créditos da linha de infra-estrutura do PRONAF. A divisão entre municípios receptores e não receptores é importante porque permite utilizar o segundo grupo como base de comparação para a estimação dos impactos da linha de

6 A questão sobre a forma ideal de análise será tratada com mais detalhes na seção 4.

7 O grupo A, formado por assentados, não foi incluído nas estimações econométricas pela inexistência de um grupo de controle para o mesmo. Assim sendo, as análises da seção 5 referem-se apenas aos grupos B, C e D.

8 A lista completa das variáveis utilizadas é apresentada na Tabela 3, anexa. 
infra-estrutura. ${ }^{9}$ Para cada entrevistado foram levantadas informações sobre o domicílio (número de habitantes, renda etc.), a produção e destino da mesma, sobre tamanho, localização e condições de acesso às propriedades, além de informações específicas sobre a participação no PRONAF. Uma lista dessas variáveis é apresentada na Tabela 3, anexa.

\section{Tabela 1 - Características PRONAF x Controle}

\begin{tabular}{lrc}
\hline Características & Controle & PRONAF \\
\hline Vínculo a sindicatos & $31 \%$ & $39 \%$ \\
Idade média (anos) & 47,9 & 47,6 \\
Reside em área urbana & $7 \%$ & $8 \%$ \\
Adota controle de pragas & $62 \%$ & $75 \%$ \\
Adota controle de erosão & $10 \%$ & $14 \%$ \\
Área média (ha) & 10,91 & 18,14 \\
Valor da produção (R\$) & $1.975,35$ & $2.473,17$ \\
Renda bruta (R\$) & $3.521,96$ & $4.014,43$ \\
\hline
\end{tabular}

Fonte: Dados da FADE/UFPE. Elaboração própria.

Uma questão importante e crucial para a análise é a escolha das variáveis a serem utilizadas para avaliar o impacto do PRONAF. Pelo objetivo do programa, pode-se inferir que se este estiver funcionando a contento, a renda, a produtividade e o emprego dos agricultores beneficiários deveriam ser maiores do que a de um grupo de indivíduos não-beneficiados. Em vista disto, deve-se fazer uso de variáveis que sejam capazes de refletir tais dimensões. Neste estudo, em particular, o impacto do PRONAF será avaliado por meio de três variáveis específicas, quais sejam: o valor da produção, o valor da produção por hectare e valor da produção por pessoa engajada na produção. Tais variáveis foram escolhidas porque são representativas de dois dos principais objetivos almejados: a) crescimento da renda, denotado pelo crescimento do valor da produção; b) crescimento na capacitação do agricultor familiar, representado pelo crescimento no valor da produção por hectare e por pessoa engajada na produção. Observe-se que, dentro de sua concepção, o PRONAF somente seria eficaz se afetasse positivamente todo este conjunto de variáveis e não apenas o valor da produção.

Vale notar que as variáveis utilizadas para a análise representam a produção total da propriedade, e não a renda monetária recebida pelo agricultor. Ou seja, a variável do valor da produção contabiliza tudo o que foi produzido em uma propriedade específica, incluindo o que foi consumido pela própria família. A justificativa para tal escolha encontra-se exatamente no baixo nível de renda dos agricultores familiares brasileiros, em que pouco, ou muito pouco, do que é produzido é efetivamente vendido para o mercado, sendo este fenômeno ainda mais forte no Nordeste do que no restante do País. Desta forma, a elevação do valor da produção pode ser entendida como um indicativo de uma melhora na qualidade de vida desses agricultores.

Ao fazer uso de variáveis do valor de produção, a análise passa a ser "menos exigente" com o programa do que o seria caso fossem levados em conta apenas os impactos sobre a renda monetária. Neste sentido, a análise dos impactos sobre a renda monetária pode ser considerada caso sejam encontrados indícios de efeitos significativos sobre o valor da produção. Entretanto, como será visto adiante, este não é o caso. O restante deste artigo é dedicado à análise dos dados levantados sobre o programa no Estado de Pernambuco.

9 Essa comparação, entretanto, não é realizada no presente trabalho, dado que o foco principal aqui é a linha de crédito direto. 


\section{AVALIANDO A EFICÁCIA DO PROGRAMA}

A análise de eficácia busca verificar se a política pública foi capaz de gerar os resultados que se esperava. O objetivo deste trabalho é, portanto, proceder à análise da eficácia do PRONAF, tomando como parâmetro as variáveis de produção apresentadas na seção 3. Antes de realizar as análises faz-se necessário, entretanto, levar em consideração os problemas técnicos que estão envolvidos nessa tarefa. As técnicas de avaliação de impacto de políticas em grupos específicos têm sido desenvolvidas para a solução do seguinte problema: como avaliar o impacto do programa em pessoas que devem encontrar-se em situações diferentes, ou seja, ser ou não beneficiado pelo programa, mas nunca nas duas simultaneamente. Ou seja, as técnicas tentam resolver o problema de avaliação com insuficiência de informações a respeito dos beneficiados.

Mais precisamente, considere-se uma pessoa $i$, uma variável de avaliação de impacto $Y$ (renda, por exemplo) e os dois estados possíveis, "1" para a situação de ter sido beneficiado e "0" para a situação de não ter sido beneficiado. Com $D=1$ indicando o primeiro estado e $D=0$, o estado alternativo, o resultado observado para a variável $Y$ da pessoa $i$ seria:

$$
Y^{i}=D Y_{1}^{i}+(1-D) Y_{0}^{i},
$$

e o ganho com o programa para o indivíduo $i$ e o ganho médio do programa poderiam ser representados, respectivamente, por $\Delta^{i}=Y_{1}^{i}-Y_{0}^{i}$ e $\Delta=E\left(\Delta^{i} \mid D=1\right)-E\left(Y_{0}^{i} \mid D=0\right)$, onde $E(\bullet \mid D=1)$ referese ao valor esperado condicionado à participação no programa.

Como não é possível observar os indivíduos nas duas situações, utiliza-se, nas avaliações, um grupo de indivíduos que não participou do programa, isto é, o grupo de controle, obtendose uma medida aproximada do impacto do programa sobre a variável considerada:

$$
\begin{aligned}
& E\left(Y_{1}^{i} \mid D=1\right)-E\left(Y_{0}^{i} \mid D=0\right)=E\left(Y_{1}^{i} \mid D=1\right)-E\left(Y_{0}^{i} \mid D=1\right)+E\left(Y_{0}^{i} \mid D=1\right)-E\left(Y_{0}^{i} \mid D=0\right) \\
& =\Delta+E\left(Y_{0}^{i} \mid D=1\right)-E\left(Y_{0}^{i} \mid D=0\right)
\end{aligned}
$$

A última diferença do lado direito da equação, diferença entre os valores esperados da variável quando da não participação no programa condicionado aos dois estados, corresponde a uma medida do erro ao se utilizar o grupo de controle. Isto deriva do fato de que a renda, por exemplo, dos indivíduos do grupo de controle não corresponde àquela dos beneficiados caso estes não tivessem participado do programa. Tal medida fornece, assim, um indicador do viés de seleção ou participação do programa, aliado ao fato de que a própria participação no programa serve, em si, para diferenciar os indivíduos (mais motivados versus menos motivados, por exemplo, já condicionando os resultados do programa).

A precisão e o grau de identificação do impacto do programa, $\Delta$, dependem, evidentemente, do tamanho do viés de seleção envolvido na avaliação. Tal magnitude, por sua vez, está vinculada ao mecanismo de seleção do grupo de controle e às técnicas de avaliação utilizadas nesta tarefa. Tais condicionantes e as técnicas utilizadas neste trabalho são discutidas a seguir. 


\subsection{Experimento social}

O que é conhecido na literatura de avaliação de impactos de políticas de programa sobre gru$\operatorname{pos}^{10}$ como Experimento Social corresponde à situação em que a escolha dos indivíduos a serem beneficiados e aqueles a pertencerem ao grupo de controle é feita aleatoriamente a partir de um grupo maior de candidatos ao programa. Em outras palavras, a distribuição dos indivíduos entre os estados $(1,0)$ é feita por meio de sorteio aleatório. Tal procedimento eliminaria qualquer viés de participação, permitindo a identificação exata do impacto do programa sobre os beneficiados $(\Delta)$.

Formalmente, na ausência de viés de participação ou seleção, ter-se-ia $E\left(Y_{0}^{i} \mid D=1\right)-E\left(Y_{0}^{i} \mid D=0\right)=0$ . Ou seja, em média, não existiriam diferenças entre a renda potencial dos não-beneficiados e daqueles que o foram, caso não tivessem recebido o programa.

\subsection{Grupo de controle não aleatório}

A alternativa à inexistência de seleção aleatória entre beneficiados e não-beneficiados implica a utilização de grupo de controle escolhido de forma não aleatória, tal como no caso da amostra utilizada no presente artigo.

De forma geral, os indivíduos deste grupo de controle são escolhidos segundo algumas características previamente elegíveis, predeterminadas, tidas como fundamentais para a dinâmica da variável-fim do programa (ser produtor familiar, ter menos de dois empregados permanentes etc.). Adicionalmente, são empregados diferentes estimadores na mensuração do impacto dos programas dos beneficiados, e que dependem do conjunto de informações disponíveis, da variável em análise e das hipóteses assumidas sobre a participação no programa.

As subseções a seguir apresentam as principais características dos estimadores utilizados neste trabalho.

\subsubsection{Diferença das médias sem controle}

A estimativa do impacto do programa sobre os beneficiados feita por meio da diferença das médias da variável-fim ou de interesse assume, arbitrariamente, que não existem diferenças expressivas entre beneficiados e grupo de controle no que diz respeito às características essenciais para a explicação do comportamento da variável de interesse. O impacto do programa sobre os beneficiados é estimado calculando-se a diferença entre as médias desta variável para os beneficiados e o grupo de controle, observando-se, então, a sua significância estatística (teste de diferenças de médias). Mais especificamente, o impacto do programa $(\Delta)$ é aproximado por:

$$
E\left(Y_{1}^{i} \mid D=1\right)-E\left(Y_{0}^{i} \mid D=0\right)=E\left(Y_{b}^{i}\right)-E\left(Y_{c}^{i}\right)
$$

onde agora $i$ se refere a indivíduos de cada grupo e $Y_{b}^{i}, Y_{c}^{i}$ dizem respeito, respectivamente, aos valores da variável para indivíduos dos grupos de beneficiados e controle. Note-se (equação (2)) que tal assunção (fortíssima) equivale, em verdade, a uma tentativa de replicar o experimento social por meio de características semelhantes dos indivíduos.

Mesmo sob a suposição, extremamente otimista, de que características importantes dos indivíduos para a variável de interesse constituam aproximações entre os dois grupos, a própria partici-

10 Para um survey acerca dessa literatura, ver, por exemplo, Friedlander, Greenberg e Robins (1997). 
pação no programa já pode sinalizar diferenciações importantes entre os indivíduos dos dois grupos e dificilmente tal estimativa fornece um valor confiável para o impacto da política.

\subsubsection{Diferenças das médias com controle}

De forma mais realista, é possível estimar o impacto do programa por meio de diferenças das médias da variável de interesse entre os dois grupos de indivíduos, reconhecendo-se, entretanto, que existem diferenças entre os indivíduos em termos de características observáveis e possivelmente não-observáveis.

Como tais características tendem a afetar tanto diretamente (operando mesmo com inexistência de programa) quanto indiretamente (motivando ou não a participação) as variáveis de interesse, um controle para tais diferenças exige, em geral, uma teoria ou hipóteses de determinação da variável de interesse e de participação no programa. O modelo econométrico abaixo, bastante geral, fornece uma representação para estimar o impacto do programa no qual as diferentes características dos grupos são consideradas, juntamente com a determinação do comportamento da variável e da participação no programa.

$$
\begin{aligned}
& Y_{i t}=\alpha_{0}+X \beta+\alpha_{1} D_{i 0}+e_{i t}, t>0 \\
& D_{i 0}=Z_{i} \gamma_{0}+u_{i 0} \\
& D_{i 0}=1,0 .
\end{aligned}
$$

A primeira equação descreve a determinação da variável de interesse do programa do indivíduo $i$ no período $t, Y_{i t}$. Nela, o vetor $X$ é composto por variáveis que supostamente explicam tal comportamento, conjuntamente com uma variável binária (dummy) que representa a participação $\left(D_{i 0}=1\right)$ ou não no programa $\left(D_{i 0}=0\right)$, decisão esta tomada no tempo 0 . A segunda equação descreve a decisão de participar ou não do programa em função das variáveis explicativas $Z$. Os termos $e_{i t}$ e $u_{i 0}$ correspondem às partes não determinísticas dos modelos e, supostamente, são bem comportados (média zero e variância constante). As demais letras correspondem a coeficientes a serem estimados.

Neste contexto, a estimativa do impacto do programa é dada por estimação pelo método de Mínimos Quadrados Ordinários (MQO) do coeficiente $\alpha_{1}$, obtido depois de se levar conta (controlar) as diferenças entre os indivíduos nas variáveis $(X)$, de determinação da variável de interesse, e nas variáveis $(Z)$, determinantes da participação. Um valor positivo e estatisticamente significante deste coeficiente indicaria, por exemplo, que o programa tem um efeito positivo sobre a variável $Y$.

Na prática, dado o desconhecimento preciso do processo de determinação de $Y$ e $D$, nem sempre é possível obter estimativas não-viesadas ou não tendenciosas do coeficiente $\alpha_{1}$. Isto é, a omissão de importantes determinantes destas variáveis ou mesmo a natureza não mensurável de outros (como é possivelmente o caso de algumas dimensões da participação) pode impedir a obtenção de estimativas que reflitam, em média, o verdadeiro valor do coeficiente procurado. O texto a seguir discute diferentes situações e possíveis soluções para a estimação.

\subsubsection{Inexistência de viés de seleção no programa}

A situação menos problemática para a estimação do coeficiente $\alpha_{1}$ corresponde àquela em que, além dos modelos das equações (4)-(6) apreenderem bem o comportamento das variáveis 
dependentes, as variáveis importantes para a determinação de $Y$ e da participação foram todas explicitamente consideradas na regressão. Em particular, não há variável que afete simultaneamente participação e $Y$ que não esteja incluída na regressão, o que também exclui a possibilidade de existência de características não-observáveis.

Nesta situação, tem-se que $E\left(D_{i 0} e_{i t}\right)=0$, não sendo relevante considerar a equação para a explicação na participação no programa. O modelo acima pode ser reduzido, então, às equações (4) e (6):

$$
\begin{aligned}
& Y_{i t}=\alpha_{0}+X \beta+\alpha_{1} D_{i 0}+e_{i t}, t>0 \\
& D_{i 0}=1,0 .
\end{aligned}
$$

Assim, para os beneficiados e os indivíduos do grupo de controle os valores da variável dependente são dados, respectivamente, por:

$$
Y_{i t}=\alpha_{0}+X \beta+\alpha_{1} D_{i 0}+e_{i t} \quad \text { e } \quad Y_{i t}=\alpha_{0}+X \beta+e_{i t} .
$$

O impacto do programa sobre a variável $Y$ pode ser então aproximado por meio da diferença entre os valores esperados da variável para os dois grupos. Tal diferença pode ser obtida mediante as estimativas de MQO dos coeficientes, uma vez que neste caso e para tal estimador ${ }^{11}$ $E(Y \mid X, D=1)=\alpha_{0}+X \beta+\alpha_{1}$ e $E(Y \mid X, D=0)=\alpha_{0}+X \beta$, tendo-se, pois:

$$
E\left(Y_{1}^{i} \mid X, D=1\right)-E\left(Y_{0}^{i} \mid X, D=0\right)=E\left(\alpha_{0}+X \beta+\alpha_{1}+e_{i t}\right)-E\left(\alpha_{0}+X \beta+e_{i t}\right)=\alpha_{1}
$$

A estimação da equação (4) permite obter então um valor para $\alpha_{1}$ e testar sua significância estatística.

\subsubsection{Existência de viés de seleção em variáveis observáveis}

Quando os participantes do programa são selecionados com base em características observáveis (agricultores etc.), estratégia comum em programas que têm público-alvo definido, há a possibilidade de que as estimativas de MQO vistas acima não forneçam resultados confiáveis, uma vez que estes podem não refletir, em média, o verdadeiro valor do parâmetro desejado (estimação viesada ou tendenciosa).

Tal possibilidade de viés ocorre se o sistema de equações (4)-(6) não considerar todas as variáveis importantes na determinação da participação que afetam também a variável de interesse. Neste caso, $E\left(D_{i 0} e_{i t}\right) \neq 0$ em virtude de que $E\left(Z_{i} e_{i t}\right) \neq 0$, onde a variável $Z_{i}$ corresponde a um determinante da participação, omitido da equação (5), que também influencia o comportamento da renda. ${ }^{12}$ Há pelo menos três soluções comumente utilizadas para solucionar tal problema, consideradas abaixo.

11 Para o estimador de MQO, $\beta$, dos coeficientes $\beta$, dada a ausência de dependência entre o termo estocástico e as variáveis explicativas, $E\left(\beta^{\prime}\right)=\beta$.

12 Para uma ilustração rápida, suponha que as mulheres sejam o público-alvo. Neste caso, a condição, por exemplo, de recém-descasada deve afetar tanto a participação quanto o comportamento da renda. A não inclusão desta condição no sistema de equações (4)-(6) pode enviesar o estimador de MQO de $\alpha_{1}$. 


\subsubsection{Inclusão das variáveis omitidas como regressores na equação}

Neste caso, a forma estimável da equação (4), já com a inclusão da variável antes omitida, seria representada por:

$$
Y_{i t}=\alpha_{0}+X \beta+\alpha_{1} D_{i 0}+Z_{i} \alpha_{2}+e_{i t}
$$

A estimação por MQO de (4)' permitiria, então, obter:

$$
E\left(Y_{1}^{i} \mid X, Z_{i}, D=1\right)-E\left(Y_{0}^{i} \mid X, Z_{i}, D=0\right)=\alpha_{1},
$$

e novamente testar a significância estatística deste coeficiente. A dificuldade, aqui, reside na mensuração destas variáveis omitidas e no conhecimento da forma funcional exata da relação entre elas e a variável de interesse, possivelmente não-linear.

\subsubsection{Matching em características observáveis}

Uma alternativa às dificuldades acima apontadas é a utilização de um estimador de matching que, ao assumir que a seleção para o programa se dá apenas a partir de características observáveis, de modo que pessoas com tais características idênticas tenham a mesma probabilidade de participação, obtém o efeito do programa considerando subgrupos de indivíduos. ${ }^{13}$

Uma vez que as pessoas com características observáveis idênticas $\left(Z_{i}\right)$ tenham a mesma probabilidade de serem escolhidas para os grupos de programa e de controle, o valor da variável de interesse $(Y)$, dadas as informações em $Z_{i}$, passa, para este grupo de pessoas, a ser estatisticamente independente do estado $D$. Mais formalmente, nesta situação ter-se-ia $\left(Y_{1}^{i}, Y_{0}^{i} \perp D_{i}\right) \mid Z_{i}$, e assim, da equação (2),

$$
E\left(Y_{0}^{i} \mid Z_{i}, D=1\right)-E\left(Y_{0}^{i} \mid Z_{i}, D=0\right)=0,
$$

onde o símbolo $\perp$ indica independência.

Desta forma, o impacto do programa $(\Delta)$ poderia ser inicialmente calculado para as pessoas com características observáveis idênticas, isto é, de acordo com a equação (2), é obtido:

$$
\Delta_{z}=E\left(Y_{1}^{i} \mid Z_{i}, D=1\right)-E\left(Y_{0}^{i} \mid Z_{i}, D=0\right),
$$

onde $\Delta_{z}$, dado pela diferença entre as médias da variável de interesse para beneficiados e controles com as mesmas características, corresponde ao impacto médio do programa nas pessoas com características $Z$ (idênticas).

O resultado médio do programa poderia, então, ser obtido por meio de uma média ponderada dos resultados para os diversos grupos, o que é feito tomando-se o valor esperado da equação (7) condicionado à situação em que $D=1$ :

$$
\Delta=E\left\{\left[E\left(Y_{1}^{i} \mid Z_{i}, D=1\right)-E\left(Y_{0}^{i} \mid Z_{i}, D=0\right)\right] \mid D=1\right\}=E\left(\Delta_{z} \mid D=1\right)
$$

13 Para uma discussão detalhada deste estimador, ver Angrist e Krueger (1999). 
Para o caso de variáveis observáveis $(Z)$ discretas, o impacto do programa pode ser representado pela soma ponderada das diferenças para cada grupo, onde os pesos são dados pela probabilidade de um indivíduo do grupo de programa possuir as características $Z$. Precisamente:

$$
\Delta=\sum_{z} \Delta_{z} \operatorname{Pr}\left(Z_{i}=Z \mid D=1\right)
$$

Em suma, o impacto do programa sobre a variável de interesse $(Y)$ é dado pela soma ponderada das diferenças entre o valor médio desta variável para beneficiados e controles para cada grupo com características idênticas, onde os pesos de cada diferença são dados pela distribuição dos beneficiados entre os grupos. Note-se, retornando à equação (2), que dadas as hipóteses aqui assumidas (grupos com características idênticas), ao menos para cada grupo é possível obter uma estimativa para $\Delta$ livre do viés de seleção em características observáveis. A dificuldade deste estimador reside, porém, na sua implementação para um número muito grande de variáveis $Z$ ou quando estas são contínuas.

\subsubsection{Propensity score}

Os métodos de Propensity Score procuram sintetizar as informações, contidas nas variáveis $Z$, que afetam a participação no programa, por meio da estimação, condicionada nestas variáveis, da probabilidade de pertencer ao grupo de beneficiados pelo programa (estimativa de propensity score). A idéia é utilizar, na equação (8), não as variáveis $Z$ diretamente, mas as probabilidades de participação de cada grupo delas derivadas. Tal procedimento resumiria as informações contidas em $Z$, fornecendo um critério único de distribuição dos indivíduos entre os grupos.

Para a aplicação do método é necessário, porém, que as propriedades estatísticas dos resultados para a variável de interesse $(Y)$ em relação a $D$ e $Z$, acima assumidas, sejam válidas também quando, ao invés de $Z$, considera-se $\operatorname{Pr}\left(Z_{i}\right)$, probabilidade de participação determinada pelas variáveis $Z_{i}$. Neste sentido, Rosenbaum e Rubin (1983) mostram que se:

$$
\left(Y_{1}^{i}, Y_{0}^{i} \perp D_{i}\right) \mid Z_{i} \text {, então }\left(Y_{1}^{i}, Y_{0}^{i} \perp D_{i}\right) \mid \operatorname{Pr}\left(Z_{i}\right) \text {, onde } \operatorname{Pr}\left(Z_{i}\right)=\operatorname{Pr}\left(D=1 \mid Z_{i}\right) \text {. }
$$

O que permite reescrever a equação (8) como:

$$
\Delta=E\left\{\left[E\left(Y_{1}^{i} \mid \operatorname{Pr}\left(Z_{i}\right), D=1\right)-E\left(Y_{0}^{i} \mid \operatorname{Pr}\left(Z_{i}\right), D=0\right)\right] \mid D=1\right\} .
$$

A obtenção de uma estimativa do impacto do programa via equação (10) é, assim, feita em dois estágios. O primeiro procura estimar as probabilidades de participações, isto é, o propensity score, para os indivíduos. Em seguida, estes são agrupados de acordo com estas probabilidades. O resultado final representa uma soma ponderada das diferenças das médias das variáveis de interesse (Y) para cada grupo, com os pesos dados pela participação dos beneficiados em cada grupo.

O expediente utilizado neste artigo seguiu aquele presente em Dehejia e Wahba (1998). Na primeira etapa, o propensity score foi estimado por meio de um modelo logit. Em seguida, a partir das probabilidades estimadas, os indivíduos da amostra foram distribuídos em cinco partes iguais. Para cada um destes grupos foram obtidas diferenças das médias da variável de interesse entre 
beneficiados e controles, sendo o impacto final do programa obtido por meio da média ponderada destas diferenças, com os pesos dados pela participação dos beneficiados em cada grupo. ${ }^{14}$ A próxima seção apresenta esses resultados.

\section{RESUlTADOS EMPÍRICOS}

$\mathrm{Na}$ Tabela 2, a seguir, são apresentadas as estimativas do impacto do programa sobre o valor da produção, o valor da produção por hectare e sobre o valor da produção por pessoa da propriedade rural para o total e para os três grupos considerados no PRONAF (grupos B, C e D) a partir dos três estimadores utilizados neste trabalho: ${ }^{15}$ a diferença das médias sem controle, a diferença das médias a partir das regressões de Mínimos Quadrados e o estimador de Propensity Score. ${ }^{16}$

Tabela 2 - Impacto do PRONAF sobre variáveis selecionadas - diferenças das médias

\begin{tabular}{|c|c|c|c|c|}
\hline$\Delta$ & Todo o Universo\# & Grupo B & Grupo C & Grupo D \\
\hline \multicolumn{5}{|l|}{ Sem Controle } \\
\hline Valor da Produção (R\$/ano) & $\begin{array}{l}500,99^{* *} \\
(123,55)\end{array}$ & $\begin{array}{l}118,59^{* *} \\
(23,69)\end{array}$ & $\begin{array}{l}519,79^{\star *} \\
(82,28)\end{array}$ & $\begin{array}{l}-2146,34^{*} \\
(1023,26)\end{array}$ \\
\hline Valor da Produção por hectare (R\$/ano) & $\begin{array}{l}-393,83^{* *} \\
(134,08)\end{array}$ & $\begin{array}{c}-121,84^{* *} \\
(22,22)\end{array}$ & $\begin{array}{l}-484,48^{*} \\
(210,48)\end{array}$ & $\begin{array}{r}-1578,30^{*} \\
(682,97)\end{array}$ \\
\hline Valor da Produção por pessoa (R\$/ano) & $\begin{array}{c}296,06^{* *} \\
(76,70) \\
\end{array}$ & $\begin{array}{c}42,67^{* *} \\
(16,21) \\
\end{array}$ & $\begin{array}{c}314,90^{* *} \\
(55,10) \\
\end{array}$ & $\begin{array}{l}-839,62 \\
(726,95) \\
\end{array}$ \\
\hline \multicolumn{5}{|l|}{ MQO } \\
\hline Valor da Produção (R\$/ano) & $\begin{array}{c}191,72^{\star} \\
(97,50)\end{array}$ & $\begin{array}{l}79,19^{* *} \\
(26,47)\end{array}$ & $\begin{array}{l}206,94^{*} \\
(87,73)\end{array}$ & $\begin{array}{l}-663,22 \\
(976,74)\end{array}$ \\
\hline Valor da Produção por hectare (R\$/ano) & $\begin{array}{l}-58,26^{* *} \\
(19,98)\end{array}$ & $\begin{array}{l}-26,52^{*} \\
(12,60)\end{array}$ & $\begin{array}{l}-55,00^{*} \\
(23,69)\end{array}$ & $\begin{array}{c}103,11 \\
(236,95)\end{array}$ \\
\hline Valor da Produção por pessoa (R\$/ano) & $\begin{array}{l}194,16^{* *} \\
(60,52)\end{array}$ & $\begin{array}{c}23,28 \\
(15,15)\end{array}$ & $\begin{array}{l}187,03^{\star *} \\
(48,40)\end{array}$ & $\begin{array}{l}-208,48 \\
(744,78)\end{array}$ \\
\hline \multicolumn{5}{|l|}{ Propensity Score } \\
\hline Valor da Produção (R\$/ano) & $\begin{array}{c}47,33 \\
(149,04)\end{array}$ & $\begin{array}{c}54,60 \\
(27,69)\end{array}$ & $\begin{array}{c}131,58 \\
(98,85)\end{array}$ & $\begin{array}{c}-458,97 \\
(1068,05)\end{array}$ \\
\hline Valor da Produção por hectare (R\$/ano) & $\begin{array}{l}-344,68^{\star} \\
(122,49)\end{array}$ & $\begin{array}{l}-80,01^{* *} \\
(18,42)\end{array}$ & $\begin{array}{l}-422,57 \\
(234,79)\end{array}$ & $\begin{array}{l}-2379,67 \\
(2015,90)\end{array}$ \\
\hline Valor da Produção por pessoa (R\$/ano) & $\begin{array}{l}108,83 \\
(88,66)\end{array}$ & $\begin{array}{c}3,00 \\
(19,91)\end{array}$ & $\begin{array}{l}143,99^{*} \\
(67,81)\end{array}$ & $\begin{array}{c}37,17 \\
(799,29)\end{array}$ \\
\hline
\end{tabular}

Obs.: Desvio padrão entre parênteses. “*” e “* *” indicam níveis de significância, respectivamente, de 5\% e 1\%. \# A amostra não inclui os assentados.

Como discutido anteriormente, os valores obtidos para as diferenças das médias sem controle são estimativas iniciais e mais grosseiras, na medida em que não consideram as diferentes características e composição dos beneficiados e não-beneficiados, e muito menos consideram a existência de viés de participação no programa, elementos que podem explicar os resultados. Já as estimativas

14 Para uma outra aplicação acerca deste método para um programa de treinamento brasileiro, ver Fernandes, Menezes-Filho e Zylberstajn (2000) e Silveira Neto (2002).

15 Como já observado, os assentados não são incluídos no estudo por falta de um grupo de controle para os mesmos.

16 A Tabela 2 apresenta apenas os resultados em termos das variáveis de interesse. As Tabelas 4 a 6 , anexas, apresentam as equações estimadas completas para o MQO e para a equação de participação. 
realizadas por meio de mínimos quadrados consideram as diferenças de características e a composição entre os dois grupos, mas ignoram, em certa medida, o fato de que os resultados podem ser explicados pelas diferenças de incentivo à participação do programa entre beneficiados e nãobeneficiados. Por fim, o estimador de Propensity Score considera explicitamente tais diferenças de incentivo ao cotejar agentes com probabilidades de participação semelhantes.

As evidências obtidas permitem destacar uma série de importantes resultados. Considerem-se, primeiramente, as estimativas para o valor da produção. Os valores obtidos da comparação sem nenhum controle (característica e participação) indicam resultado positivo do programa (beneficiado, em média, com valor da produção $\mathrm{R} \$$ 500,99 acima do valor da produção dos não-beneficiados), mas note-se que tal resultado, para todos os grupos e para o universo, é totalmente explicado pelas diferentes características dos agentes e pelas diferentes probabilidades de participação: as diferenças diminuem quando é considerado o estimador de Mínimos Quadrados e desaparecem quando se considera o estimador de Propensity Score. Desta forma, as evidências indicam que o programa não foi eficaz quanto aos seus efeitos sobre o valor da produção, nem para todo o universo, nem para qualquer dos grupos considerados para o período investigado.

Para estimativas do impacto do programa sobre o valor da produção por hectare, as evidências apontam, quando todo o universo é considerado, um efeito negativo do programa, presente nas evidências geradas por todos os três estimadores (para o estimador de Propensity Score o diferencial negativo fica em torno de $\mathrm{R} \$ 345,00)$. Quando se consideram as evidências por grupos, apenas as propriedades rurais do grupo D (com maiores valores de renda domiciliar), nas suas estimativas com controles, não apresentam o resultado negativo obtido na agregação. Tal resultado sugere que o programa tem incentivado uma ocupação mais extensiva da propriedade sem, contudo, ter havido elevações no mesmo ritmo para a produção, evidenciando, assim, perda de produtividade.

Por fim, os resultados obtidos para o valor da produção por pessoa, de forma geral, seguem o padrão obtido para aqueles do valor da produção: embora positivos, diminuem de valor à medida que mais controles são introduzidos nas estimativas. A consideração das estimativas por grupos de beneficiados permite, todavia, perceber algumas importantes diferenças.

Para o grupo B (propriedades de menor valor da produção), as evidências seguem o comportamento já observado para todo o universo considerado, ou seja, o impacto positivo observado do programa diminui, deixando de ser significante já a partir das estimações com mínimos quadrados. Já para as propriedades rurais consideradas no grupo C, embora também os valores positivos apresentem trajetória descendente, estes se mantêm significantes mesmo após serem consideradas as diferenças de características e probabilidades de participações no programa. Tal resultado, em vista da não significância do impacto do programa sobre o valor da produção, não seria imediatamente esperado e requer maior aprofundamento da investigação. Algumas possibilidades deverão ser exploradas em outros estudos. Uma delas é de que tal resultado possa estar refletindo um potencial efeito "vizinhança" ou parentesco: indivíduos inicialmente com residências em propriedades próximas e que são beneficiados pelo programa teriam informações e incentivo para o cultivo em novas áreas próprias, deixando as propriedades de trabalho originais.

Tal resultado também, ao menos parcial e potencialmente, pode ser explicado pela presença de viés de seleção, não controlado pelos estimadores utilizados, que fariam os indivíduos das propriedades beneficiadas, mais empreendedores e dinâmicos, reagirem de forma mais decidida em face do modesto resultado do programa em termos de valor da produção. Nesta perspectiva, tais indivíduos atuariam no sentido de obter alternativas de renda, deixando a propriedade com menor número de pessoas engajadas na produção. Para o último grupo considerado (grupo D), as evidên- 
cias indicam que o programa foi ineficaz no sentido de elevar o valor da produção por pessoa, o que parece confirmar os modestos resultados do programa para este grupo.

\section{CONCLUSÕES}

A introdução do PRONAF em meados da década de 1990 representou um avanço significativo em termos concepção dos programas de apoio ao pequeno agricultor no Brasil. O principal elemento de ruptura com os programas de crédito anteriores foi a ênfase no crescimento da capacidade produtiva a partir do suporte à capacitação tecnológica, assistência técnica e infra-estrutura. Tal ênfase introduziu elementos focais na análise de crédito até então inexistentes. Em linhas gerais, nos programas anteriores ao PRONAF o agricultor era visto per se pelo agente financeiro, tendo a estrutura de concessão de crédito do PRONAF instituído, ainda que de maneira ainda pouco efetiva, a figura da cadeia produtiva.

Os resultados encontrados nesta avaliação, baseados em dados primários obtidos em pesquisa ampla com os agentes recipientes, e utilizando técnicas para controlar o viés de participação, sugerem que o impacto do PRONAF para a renda e a produtividade dos seus beneficiários foi bastante reduzido e mesmo negativo em alguns casos, considerando o período de análise. Neste sentido, este resultado encontrado para o Estado de Pernambuco é condizente com aqueles encontrados em outros estudos sobre o programa, à exceção daqueles realizados para o Sul do Brasil.

Tais resultados contraditórios sugerem que pode haver um conflito enfrentado por um programa voltado para agricultores familiares que têm ou podem vir a ter participação nos mercados, mas que também objetiva políticas sociais sobre todo o grupo de agricultores familiares. O viés de seleção, os problemas de efetividade e os efeitos reduzidos na renda/produtividade em Estados onde a agricultura familiar não é integrada à agroindústria aqui verificados, vistos à luz de outras avaliações, sugerem que o programa pode estar obtendo êxito somente em localidades onde esta integração esteja já estabelecida, com resultados tímidos nas demais.

Verifica-se, atualmente, que algumas modificações, em termos dos critérios de concessão de créditos, avaliação dos agricultores, modalidades e tetos de financiamento, foram postas em prática a partir de 2003. Tais modificações elevaram o número de contratos e permitiram que, pela primeira vez, o orçamento fosse inteiramente utilizado. No entanto, ainda não é possível afirmar se tais modificações levarão a que o programa se torne, de fato, efetivo.

\section{REFERÊNCIAS}

Angrist, E. J.; Krueger, A. B. Empirical strategies in labor economics. In: Ashenfelter, O.; Card, D., Handbook of labor economics, v. 3, 1999.

Cardoso, A.; Ortega, A. Uma avaliação do PRONAF à luz da experiência européia de desenvolvimento rural, o programa leader. Anais do XXXVIII Encontro da SOBER. 2000.

Couto Filho, V.; Cerqueira, P. Um olhar sobre o crédito do PRONAF na Bahia. Bahia Agrícola, v. 5, n. 2, novembro de 2002.

Dehejia, R. H.; Wahba, S. Causal effects in non-experimental studies: re-evaluating the evaluation of training programs. NBER Working Paper 6586, 1998.

FAO/INCRA. O novo retrato da agricultura familiar: o Brasil redescoberto. Projeto de Cooperação Técnica INCRA/FAO. Em www.desenvolvimentoagrario.gov.br. 2000. 
FECAMP. Estudos-de-caso em campo para avaliação dos impactos do PRONAF. Convênio PCT/IICA-PRONAF e Fundação de Economia de Campinas - FECAMP, 2002.

Feijó, R. Uma avaliação preliminar do PRONAF na produtividade da agricultura familiar. Textos para discussão - Série Economia. TD-E/ 37 - 2003. Universidade de São Paulo, Faculdade de Administração, Economia e Contabilidade de Ribeirão Preto, 2003.

Fernandes, R.; Menezes-Filho, N.; Zylberstajn, H. Uma avaliação do impacto do programa de treinamento do Sindicato dos Metalúrgicos de São Paulo, 2000. Mimeografado.

Friendlander, D.; Greenberg, D. H.; Robins, P. K. Evaluating government training programs for economically disadvantaged. Journal of Economic Perspectives, v. XXXV, December 1997.

Rosenbaum, P. R.; Rubin, D. B. The Central role of the propensity score in observational studies for causal effects. Biometrika, v. 70, p. 41-55, 1983.

Silveira-Neto, R. Eficácia e viés de seleção em programas de qualificação em trabalhadores em situação economicamente desvantajosa: evidências para o Estado de Pernambuco. Revista Econômica do Nordeste, v. 4, n. , p. 1-22, 2002.

\section{ANEXOS}

Tabela 3 - Lista de variáveis utilizadas na determinação do impacto do PRONAF

\begin{tabular}{|c|c|c|}
\hline Variáveis & siglas & Categoria \\
\hline Valor da produção nos últimos 12 meses & vprod & Produção \\
\hline Valor da produção por hectare nos últimos 12 meses & Vpha & Produção \\
\hline Valor da produção por pessoa engajada nos últimos 12 meses & vppess & Produção \\
\hline Dummy para indivíduos integrados à agroindústria & AGIND & Propriedade \\
\hline Dummy para indivíduos que estão afiliados a cooperativas & COOP & Propriedade \\
\hline Anos de experiência do chefe do domicílio & EXPERI & Domiciliar \\
\hline Anos de estudo do chefe do domicílio & EST_CH & Domiciliar \\
\hline Anos de estudo do cônjuge & EST_CJ & Domiciliar \\
\hline Área da propriedade & AREA & Propriedade \\
\hline Homens dia de trabalho & HODIA & Produção \\
\hline Gastos com insumo para a produção nos últimos 12 meses & INSUMO & Produção \\
\hline \multirow{3}{*}{$\begin{array}{l}\text { Porcentual da produção proveniente da agricultura nos últimos } 12 \text { meses } \\
\text { Número de visitas de técnicos de assistência técnica na última safra nos últimos } 12 \\
\text { meses } \\
\text { Horas freqüentadas em cursos agrícolas }\end{array}$} & AGRICULT & Produção \\
\hline & AT_GER & Domiciliar \\
\hline & CP_GER & Domiciliar \\
\hline Dummy para localização da propriedade (área urbana = 1) & URBANA & Propriedade \\
\hline Número de residentes no domicílio & RESIDE & Domiciliar \\
\hline Número de aposentados no domicílio & APOSEN & Domiciliar \\
\hline Dummy para uso de controles de praga (se utiliza $=1$ ) & PRAGA & Produção \\
\hline Porcentual da produção que recebe irrigação nos últimos 12 meses & IRRIG & Produção \\
\hline Dummy para uso de energia elétrica na propriedade (se utiliza =1) & ENERG & Produção \\
\hline $\begin{array}{l}\text { Dummy para recebimento de outros créditos além dos recursos do PRONAF (se } \\
\text { recebeu =1) }\end{array}$ & OUTCRE & Crédito \\
\hline Distância em Km do centro de distribuição dos produtos & DST_CD & Propriedade \\
\hline Porcentual da distância que é asfaltada & ASF_CD & Propriedade \\
\hline Dummy para recebimento de crédito do PRONAF (se recebeu =1) & PRONAF & Crédito \\
\hline Renda municipal em 2000 & RENDA_MU & Municipal \\
\hline Pluviometria média municipal em 2000 & CHUVA_LN & Municipal \\
\hline Variância da pluviometria & VAR_CHUV & Municipal \\
\hline
\end{tabular}


Tabela 4 - Resultados das regressões por mínimos quadrados ordinários

\begin{tabular}{|c|c|c|c|c|c|c|c|c|c|c|c|c|}
\hline \multirow[b]{2}{*}{ Variáveis } & \multicolumn{4}{|c|}{ VPROD } & \multicolumn{4}{|c|}{ VPHA } & \multicolumn{4}{|c|}{ VPRDPESS } \\
\hline & Total & Grupo B & Grupo C & Grupo D & Total & Grupo B & Grupo C & Grupo D & Total & Grupo B & Grupo C & Grupo D \\
\hline \multirow[t]{2}{*}{ C } & $-6125,33^{* *}$ & $-989,43^{*}$ & $-2753,97^{*}$ & $-13446,87$ & $-1472,84^{* *}$ & $-344,06$ & $-1197,16^{* *}$ & & $-2869,27^{* *}$ & $-364,45$ & $-600,22$ & $-13072,92$ \\
\hline & $(1460,91)$ & $(441,36)$ & $(1236,47)$ & $(15659,85)$ & $(316,46)$ & $(280,73)$ & $(319,25)$ & $(2586,67)$ & $(891,00)$ & $(272,73)$ & $(732,47)$ & $(8835,71)$ \\
\hline \multirow[t]{2}{*}{ AGIND } & $935,91^{\text {** }}$ & 105,03 & $482,67^{*}$ & 2661,25 & $165,29^{*}$ & 28,13 & 105,46 & $-444,22$ & 268,93 & 48,31 & 233,29 & 860,02 \\
\hline & $(328,75)$ & $(111,01)$ & $(235,46)$ & $(2203,39)$ & $(69,33)$ & $(42,20)$ & $(73,83)$ & & $(186,04)$ & $(61,70)$ & $(124,54)$ & $(1145,53)$ \\
\hline \multirow[t]{2}{*}{$\mathrm{COOP}$} & $987,50^{* *}$ & 136,28 & $553,29^{* *}$ & 584,42 & $164,15^{* *}$ & 14,88 & $126,34^{* *}$ & $-480,30^{*}$ & $373,63^{*}$ & 51,54 & 16,69 & $-478,28$ \\
\hline & $(246,38)$ & $(74,86)$ & $(187,98)$ & $(1636,82)$ & $(46,75)$ & $(24,60)$ & $(51,16)$ & $(244,18)$ & $(159,94)$ & $(38,97)$ & $(99,67)$ & $(899,79)$ \\
\hline \multirow[t]{2}{*}{ EXPERI } & $34,63^{* *}$ & 3,41 & 0,99 & & $-1,86$ & $-0,04$ & & & 5,98 & 0,46 & $-14,75$ & $-90,59$ \\
\hline & $(11,12)$ & $(4,26)$ & $(10,28)$ & $(195,16)$ & $(3,02)$ & $(2,10)$ & & & & $(2,14)$ & $(7,85)$ & $(122,48)$ \\
\hline \multirow[t]{2}{*}{$($ EXPERI)^2 } & $-0,28$ & $-0,03$ & 0,13 & 2,38 & 0,02 & 0,00 & 0,05 & 0,27 & $-0,05$ & $-0,02$ & 0,19 & 1,00 \\
\hline & $(0,15)$ & $(0,05)$ & $(0,14)$ & $(2,62)$ & $(0,04)$ & $(0,03)$ & $(0,05)$ & $(0,44)$ & $(0,10)$ & $(0,03)$ & $(0,10)$ & $(1,56)$ \\
\hline \multirow[t]{2}{*}{ EST_CH } & $47,72^{*}$ & 7,28 & 17,03 & 39,00 & $-1,08$ & $-2,25$ & $-4,65$ & $-41,19$ & $35,48^{*}$ & 1,17 & 14,81 & 61,49 \\
\hline & $(23,07)$ & $(7,63)$ & $(17,07)$ & $(149,98)$ & $(4,40)$ & $(4,07)$ & & & & $(4,18)$ & & $(131,45)$ \\
\hline \multirow[t]{2}{*}{ EST_CJ } & $42,54^{*}$ & 11,08 & 3,78 & $-290,04$ & $-1,24$ & 1,58 & $-1,72$ & $-32,25$ & $15^{* *}$ & 6,82 & 12,97 & $-19,24$ \\
\hline & $(19,30)$ & $(6,07)$ & $(14,11)$ & $(153,47)$ & $(3,47)$ & $(2,69)$ & $(3,74)$ & $(30,54)$ & (13, & $(3,63)$ & $(9,00)$ & $(112,61)$ \\
\hline \multirow[t]{2}{*}{ AREA } & $17,35^{\star *}$ & 1,10 & $11,15^{\star *}$ & $42,91^{* *}$ & & & & & $9,01^{* *}$ & 1,08 & $4,65^{\star *}$ & 25,45 \\
\hline & $(3,73)$ & $(0,85)$ & $(2,40)$ & $(13,96)$ & & & & & & $(0,67)$ & $(1,67)$ & $(15,93)$ \\
\hline \multirow[t]{2}{*}{ HODIA } & $-0,32^{* *}$ & $-0,05^{\star *}$ & $-0,42^{* *}$ & $-8,73^{\star *}$ & $0,12^{\star *}$ & $0,09^{*}$ & $0,23^{\text {** }}$ & $1,71^{* *}$ & & & & \\
\hline & $(0,06)$ & $(0,01)$ & $(0,10)$ & $(3,04)$ & $(0,03)$ & $(0,04)$ & $(0,06)$ & $(0,23)$ & & & & \\
\hline INSUMO & $0,25^{\star *}$ & 0,02 & $0,26^{* *}$ & $1,07^{\star *}$ & $0,20^{* *}$ & $0,08^{* *}$ & $0,21^{* *}$ & $4,66^{* *}$ & 0,05 & 0,00 & 0,02 & 0,16 \\
\hline & $(0,07)$ & $(0,03)$ & $(0,10)$ & $(0,34)$ & $(0,05)$ & $(0,02)$ & $(0,07)$ & $(1,66)$ & $(0,05)$ & $(0,01)$ & $(0,04)$ & $(0,13)$ \\
\hline AGRICULT & $-11,05^{\star *}$ & $-3,89^{* *}$ & $-7,82^{* *}$ & 30,42 & $-0,77^{\star *}$ & $-1,07^{\star *}$ & $-0,42$ & 3,69 & $-4,5$ & $-1,92^{\star *}$ & $-3,73^{\star *}$ & 10,11 \\
\hline & $(1,39)$ & $(0,44)$ & $(1,26)$ & & & $(0,25)$ & & & & $(0,27)$ & & $(10,84)$ \\
\hline AT_GER & 1,65 & $2,78^{*}$ & $-0,34$ & $-52,10$ & $0,98^{*}$ & 0,73 & $0,60^{* *}$ & $-10,18$ & 2,48 & 0,34 & 0,19 & $-4,32$ \\
\hline & $(1,90)$ & $(1,30)$ & $(0,88)$ & $(36,89)$ & $(0,43)$ & $(1,28)$ & $(0,23)$ & $(8,60)$ & $(1,93)$ & $(0,71)$ & $(0,67)$ & $(21,76)$ \\
\hline CP_GER & 0,97 & $-1,25$ & 0,55 & 1,08 & 0,03 & $-0,65$ & 0,00 & -0, & 0,0 & $-0,54$ & 0,08 & $-4,26$ \\
\hline & & $(0,92)$ & $(0,53)$ & $(17,8$ & & & & & & $(0,44)$ & & $(11,19)$ \\
\hline URBANA & 405,68 & $-68,93$ & $-114,23$ & 696,91 & $-1,44$ & $-23,59$ & $-23,33$ & 292,85 & $369,53^{*}$ & $-26,91$ & 15,17 & 13,61 \\
\hline & $(235,94)$ & $(49,55)$ & $(160,51)$ & $(1598,11)$ & $(44,61)$ & $(22,46)$ & $(46,08)$ & $(248,52)$ & (151, & $(26,00)$ & $(107,97)$ & $(1210,48)$ \\
\hline RESIDE & $-19,13$ & $13,76^{*}$ & $-29,32$ & $-346,29$ & $-6,49$ & $-5,13$ & $-10,00^{*}$ & $-70,59$ & $-91,90^{* *}$ & $-12,29^{* *}$ & $-104,68^{* *}$ & $-642,63^{\star \star}$ \\
\hline & $(19,03)$ & $(6,22)$ & $(17,85)$ & $(232,85)$ & $(4,23)$ & $(3,12)$ & $(5,01)$ & $(53,74)$ & $(12,28)$ & $(3,61)$ & $(8,68)$ & $(138,89)$ \\
\hline APOSEN & $-72,24$ & 32,04 & 45,02 & $-908,54$ & $-38,14^{*}$ & $-4,61$ & $-31,98$ & $-262,07$ & $-18,96$ & 22,64 & 59,46 & $-619,28$ \\
\hline & $(74,54)$ & $(22,69)$ & $(68,69)$ & $(875,68)$ & $(17,05)$ & $(11,33)$ & $(19,83)$ & & $(48,27)$ & $(13,54)$ & & $(573,71)$ \\
\hline PRAGA & $421,29^{* *}$ & $95,54^{* *}$ & $399,79^{* *}$ & 323,18 & 14,13 & $-2,41$ & 25,58 & $-58,53$ & $319,60^{* *}$ & $33,19^{*}$ & $233,88^{* *}$ & 411,23 \\
\hline & $(94,49)$ & $(27,61)$ & $(92,76)$ & $(1372,57)$ & $(23,24)$ & $(15,59)$ & $(27,30)$ & $(319,66)$ & $(56,17)$ & $(15,13)$ & $(48,22)$ & $(911,17)$ \\
\hline IRRIG & $20,20^{* *}$ & 1,10 & $13,21^{* *}$ & 15,56 & $6,81^{* *}$ & 0,49 & $5,98^{* *}$ & $-0,04$ & 12,1 & 0,27 & $6,61^{* *}$ & 8,95 \\
\hline & & & & & & & & & & & & $(9,74)$ \\
\hline ENERG & $966,67^{\star *}$ & 121,56 & $513,80^{* *}$ & $-945,55$ & 83,04 & 3,84 & 41,45 & $-14,85$ & $550,80^{* *}$ & 63,79 & 174,84 & $-1227,94$ \\
\hline & $(228,28)$ & $(81,19)$ & $(169,20)$ & $(1098,30)$ & $(46,90)$ & $(33,28)$ & $(47,67)$ & $(268,91)$ & $(152,45)$ & $(43,94)$ & $(94,59)$ & $(824,73)$ \\
\hline OUTCRE & 379,29 & 79,90 & 123,48 & 291,27 & 1,30 & 19,17 & $-27,25$ & $-301,46$ & 93,24 & 47,57 & $-10,59$ & 1143,68 \\
\hline & $(211,69)$ & $(60,28)$ & $(162,86)$ & $(1441,09)$ & $(37,91)$ & $(38,49)$ & & $(275,98)$ & $(129,36)$ & $(32,39)$ & $(86,43)$ & $(1079,67)$ \\
\hline DST_CD & $-5,25$ & 0,48 & 1,08 & $-67,86$ & $-2,52^{\star *}$ & $-1,06^{*}$ & $-1,79^{*}$ & $-4,11$ & $-3,12$ & $-0,61$ & 1,89 & $-48,09$ \\
\hline & $(4,40)$ & & & & & & & & & $(0,67)$ & & $(32,52)$ \\
\hline ASF_CD & 2,28 & $0,99^{* *}$ & 0,61 & 6,28 & $0,89^{* *}$ & 0,38 & 0,63 & 2,08 & 1,01 & 0,29 & 0,57 & 8,50 \\
\hline & $(1,30)$ & & $(1,19)$ & $(14,27)$ & & $(0,20)$ & $(0,36)$ & & & $(0,22)$ & $(0,65)$ & $(10,80)$ \\
\hline PRONAF & $191,72^{*}$ & $79,19^{* *}$ & $206,94^{*}$ & $-663,22$ & $-58,27^{* *}$ & $-26,52^{*}$ & $-55,00^{*}$ & 103,11 & $194,16^{* *}$ & 23,28 & $187,03^{* *}$ & $-208,48$ \\
\hline & $(97,50)$ & $(26,47)$ & & & & & $(23,69)$ & & & $(15,15)$ & $(48,41)$ & $(744,78)$ \\
\hline RENDA_MU & $163,35^{* *}$ & $35,17^{\star}$ & 48,38 & $1287,86^{* *}$ & $41,73^{* *}$ & $22,71^{* *}$ & 16,43 & $-68,97$ & $111,61^{* *}$ & $21,24^{\star *}$ & 20,32 & $749,79^{*}$ \\
\hline & $(52,15)$ & $(14,65)$ & $(44,82)$ & $(17740)$ & $(10,37)$ & $(7,20)$ & $(9,88)$ & & $(32,63)$ & $(7,90)$ & $(25,09)$ & $(339,77)$ \\
\hline CHUVA_LN & 242,34 & $-66,53$ & $-7,30$ & $-11351,40$ & $610,62^{* *}$ & $305,10^{* *}$ & $620,89^{* *}$ & $-848,29$ & $-71,17$ & $-165,91^{*}$ & $-412,06$ & $-8054,22^{*}$ \\
\hline & & $(153,41)$ & & & & & & & & $(81,68)$ & & $(3772,55)$ \\
\hline VAR_CHUV & 774,65 & 265,42 & 876,44 & 12621,06 & $-293,61^{*}$ & $-236,59^{*}$ & $-262,96$ & 1010,20 & 511,97 & $249,39^{*}$ & $806,20^{*}$ & $10240,79^{*}$ \\
\hline & $(682,36)$ & $(198,87)$ & $(646,43)$ & $(8027,17)$ & $(145,41)$ & $(110,70)$ & $(159,34)$ & $(1440,48)$ & $(420,23)$ & $(111,70)$ & $(362,11)$ & $(4816,63)$ \\
\hline $\mathrm{R}^{2}$ & 0,64 & 0,24 & 0,21 & 0,27 & 0,90 & 0,80 & 0,85 & 0,90 & 0,59 & 0,53 & 0,50 & 0,39 \\
\hline $\mathrm{R}^{2}$ ajustado & 0,63 & 0,21 & 0,20 & & 0,90 & 0,79 & 0,85 & 0,89 & 0,58 & 0,50 & 0,49 & 0,31 \\
\hline Estatística-F & 70 & 10,06 & 14,34 & 3,07 & 320,34 & 70,61 & 153,14 & 76,51 & 82,42 & 16,40 & 31,37 & 4,84 \\
\hline $\operatorname{Prob}(\mathrm{F})$ & 0 & 0 & 0 & 0 & 0 & 0 & 0 & 0 & 0 & 0 & & 0 \\
\hline Observações & 3132 & 831 & 2056 & 245 & 3132 & 831 & 2056 & 245 & 3127 & 830 & 2053 & 244 \\
\hline
\end{tabular}

Obs.: Desvio padrão entre parênteses. “*” e “**” indicam níveis de significância, respectivamente, de $5 \%$ e $1 \%$. 
Tabela 5 - Modelo Logit para estimação da probabilidade de ser beneficiado pelo PRONAF

\begin{tabular}{|c|c|c|c|c|}
\hline Variáveis independentes & Total & Grupo B & Grupo C & Grupo D \\
\hline ASSOCIA & $\begin{array}{l}-0.20 \\
(0.21)\end{array}$ & $\begin{array}{c}0.50 \\
(0.49)\end{array}$ & $\begin{array}{l}-0.53^{*} \\
(0.27)\end{array}$ & $\begin{array}{c}0.46 \\
(0.56)\end{array}$ \\
\hline SIND & $\begin{array}{l}-0.02 \\
(0.24)\end{array}$ & $\begin{array}{c}0.27 \\
(0.53)\end{array}$ & $\begin{array}{l}-0.18 \\
(0.3)\end{array}$ & $\begin{array}{c}0.20 \\
(0.71)\end{array}$ \\
\hline ASSOCOOP & $\begin{array}{c}0.12 \\
(0.30)\end{array}$ & $\begin{array}{l}-0.18 \\
(0.71)\end{array}$ & $\begin{array}{c}0.25 \\
(0.39)\end{array}$ & $\begin{array}{l}-0.75 \\
(0.86)\end{array}$ \\
\hline INTEGRAC & $\begin{array}{c}0.38^{* *} \\
(0.15)\end{array}$ & $\begin{array}{c}0.34 \\
(0.26)\end{array}$ & $\begin{array}{c}0.41^{*} \\
(0.19)\end{array}$ & $\begin{array}{c}0.27 \\
(0.62)\end{array}$ \\
\hline CAPSOCIA & $\begin{array}{c}0.46^{*} \\
(0.21)\end{array}$ & $\begin{array}{c}0.17 \\
(0.47)\end{array}$ & $\begin{array}{c}0.58^{*} \\
(0.26)\end{array}$ & $\begin{array}{l}0.6 \\
(0.59)\end{array}$ \\
\hline DIRRI & $\begin{array}{c}0.13 \\
(0.08)\end{array}$ & $\begin{array}{c}0.16 \\
(0.15)\end{array}$ & $\begin{array}{c}0.02 \\
(0.11)\end{array}$ & $\begin{array}{c}0.39 \\
(0.31)\end{array}$ \\
\hline DID1825 & $\begin{array}{l}-0.31 \\
(0.19)\end{array}$ & $\begin{array}{c}0.15 \\
(0.36)\end{array}$ & $\begin{array}{l}-0.55^{*} \\
(0.24)\end{array}$ & $\begin{array}{l}-0.01 \\
(1.08)\end{array}$ \\
\hline DID2635 & $\begin{array}{l}-0.24^{*} \\
(0.11)\end{array}$ & $\begin{array}{c}0.15 \\
(0.20)\end{array}$ & $\begin{array}{l}-0.41^{\text {** }} \\
(0.13)\end{array}$ & $\begin{array}{l}-0.53 \\
(0.49)\end{array}$ \\
\hline DID3645 & $\begin{array}{l}-0.07 \\
(0.09)\end{array}$ & $\begin{array}{l}-0.03 \\
(0.18)\end{array}$ & $\begin{array}{l}-0.09 \\
(0.11)\end{array}$ & $\begin{array}{c}0.10 \\
(0.39)\end{array}$ \\
\hline DED58 & $\begin{array}{c}0.21 \\
(0.13)\end{array}$ & $\begin{array}{c}0.23 \\
(0.30)\end{array}$ & $\begin{array}{c}0.13 \\
(0.16)\end{array}$ & $\begin{array}{c}0.97^{*} \\
(0.49)\end{array}$ \\
\hline DED811 & $\begin{array}{c}0.38 \\
(0.23)\end{array}$ & $\begin{array}{l}-0.09 \\
(0.72)\end{array}$ & $\begin{array}{c}0.46 \\
(0.28)\end{array}$ & $\begin{array}{c}0.53 \\
(0.57)\end{array}$ \\
\hline DED12 & $\begin{array}{l}-0.19 \\
(0.95)\end{array}$ & $0.00^{* *}$ & $0.00^{* *}$ & $0.00^{* *}$ \\
\hline DAT_GER & $\begin{array}{l}-0.73^{* *} \\
(0.13)\end{array}$ & $\begin{array}{l}-0.86^{* *} \\
(0.34)\end{array}$ & $\begin{array}{l}-0.71^{\text {** }} \\
(0.16)\end{array}$ & $\begin{array}{l}-0.77 \\
(0.41)\end{array}$ \\
\hline DCP_GER & $\begin{array}{l}-0.50^{\star *} \\
(0.12)\end{array}$ & $\begin{array}{l}-0.83^{\star *} \\
(0.25)\end{array}$ & $\begin{array}{l}-0.46^{* *} \\
(0.14)\end{array}$ & $\begin{array}{l}-0.25 \\
(0.39)\end{array}$ \\
\hline URBANA & $\begin{array}{l}-0.29^{*} \\
(0.15)\end{array}$ & $\begin{array}{l}-0.64^{*} \\
(0.29)\end{array}$ & $\begin{array}{l}-0.17 \\
(0.19)\end{array}$ & $\begin{array}{c}0.20 \\
(0.47)\end{array}$ \\
\hline DAPOSE & $\begin{array}{c}0.38^{* *} \\
(0.08)\end{array}$ & $\begin{array}{c}0.24 \\
(0.16)\end{array}$ & $\begin{array}{c}0.39^{* *} \\
(0.10)\end{array}$ & $\begin{array}{c}0.64^{*} \\
(0.32)\end{array}$ \\
\hline PRAGA & $\begin{array}{c}0.52^{* *} \\
(0.08)\end{array}$ & $\begin{array}{c}0.69^{* *} \\
(0.14)\end{array}$ & $\begin{array}{c}0.44^{* *} \\
(0.10)\end{array}$ & $\begin{array}{l}-0.17 \\
(0.40)\end{array}$ \\
\hline ENERG & $\begin{array}{c}0.06 \\
(0.12)\end{array}$ & $\begin{array}{c}0.09 \\
(0.34)\end{array}$ & $\begin{array}{c}0.06 \\
(0.16)\end{array}$ & $\begin{array}{c}-0.13 \\
(0.33)\end{array}$ \\
\hline OUTCRE & $\begin{array}{c}0.36^{* *} \\
(0.14)\end{array}$ & $\begin{array}{c}0.50 \\
(0.31)\end{array}$ & $\begin{array}{c}0.26 \\
(0.17)\end{array}$ & $\begin{array}{c}0.63 \\
(0.43)\end{array}$ \\
\hline DAREA & $\begin{array}{c}0.90^{* *} \\
(0.09)\end{array}$ & $\begin{array}{l}0.71^{\text {** }} \\
(0.17)\end{array}$ & $\begin{array}{c}0.98^{* *} \\
(0.11)\end{array}$ & $\begin{array}{c}0.88^{* *} \\
(0.31)\end{array}$ \\
\hline DREMUN & $\begin{array}{l}-0.32^{* *} \\
(0.07)\end{array}$ & $\begin{array}{l}-0.23 \\
(0.13)\end{array}$ & $\begin{array}{l}-0.30^{* *} \\
(0.09)\end{array}$ & $\begin{array}{l}-1.07^{* \star} \\
(0.30)\end{array}$ \\
\hline _CONS & $\begin{array}{l}-0.12 \\
(0.21)\end{array}$ & $\begin{array}{c}0.13 \\
(0.47)\end{array}$ & $\begin{array}{c}0.01 \\
(0.26)\end{array}$ & $\begin{array}{c}0.22 \\
(0.74)\end{array}$ \\
\hline chi2(21) & 613.3 & 183.48 & 397.29 & 57.94 \\
\hline Prob > chi2 & 0.00 & 0.00 & 0.00 & 0.00 \\
\hline Observações & 3798 & 1145 & 2380 & 268 \\
\hline
\end{tabular}

Obs.: Desvio padrão entre parênteses. “*” e “* *” indicam níveis de significância, respectivamente, de 5\% e 1\%. 
Tabela 6 - Lista de variáveis utilizadas para probabilidade de participação (dummies)

\begin{tabular}{|c|c|c|c|}
\hline Variáveis & Siglas & Variáveis & Siglas \\
\hline $\begin{array}{l}\text { Indivíduos que estão associados a associações de } \\
\text { produtores }\end{array}$ & associa & Grupo com 12 o mais anos de estudo & $\operatorname{ded} 12$ \\
\hline Indivíduos que estão associados a sindicatos & sind & Assistência técnica na última safra & $\begin{array}{l}\text { dat_ } \\
\text { ger }\end{array}$ \\
\hline Indivíduos que estão associados a cooperativas & assocoop & Horas freqüentadas em cursos agrícolas & $\begin{array}{l}\text { dcp_ } \\
\text { ger }\end{array}$ \\
\hline Indivíduos que estão associados a agroindústria & integrac & Localização da propriedade & urbana \\
\hline Capital social & capsocia & Indivíduos aposentados & dapose \\
\hline $\begin{array}{l}\text { Produção que recebeu irrigação nos últimos } 12 \\
\text { meses }\end{array}$ & dirri & Uso de controle de praga & praga \\
\hline Grupo com idade entre 18 e 25 anos de idade & $\operatorname{did} 1825$ & Uso de energia elétrica na propriedade & energ \\
\hline Grupo com idade entre 26 e 35 anos de idade & $\operatorname{did} 2635$ & $\begin{array}{l}\text { Recebimento de outros créditos além dos } \\
\text { recursos do PRONAF }\end{array}$ & outcre \\
\hline Grupo com idade entre 36 e 45 anos de idade & $\operatorname{did} 3645$ & Área > 15 hectare & darea \\
\hline Grupo com 5 a 8 anos de estudo & $\operatorname{ded} 58$ & Renda municipal > 17 & $\begin{array}{l}\text { dre- } \\
\text { mun }\end{array}$ \\
\hline Grupo com 8 a 11 anos de estudo & ded811 & Constante & _cons \\
\hline
\end{tabular}

